ORNL/TM-2016/331

CRADA/NFE-15-05761

\title{
Additive Manufacturing of Isotropic NdFeB Bonded Permanent Magnets
}

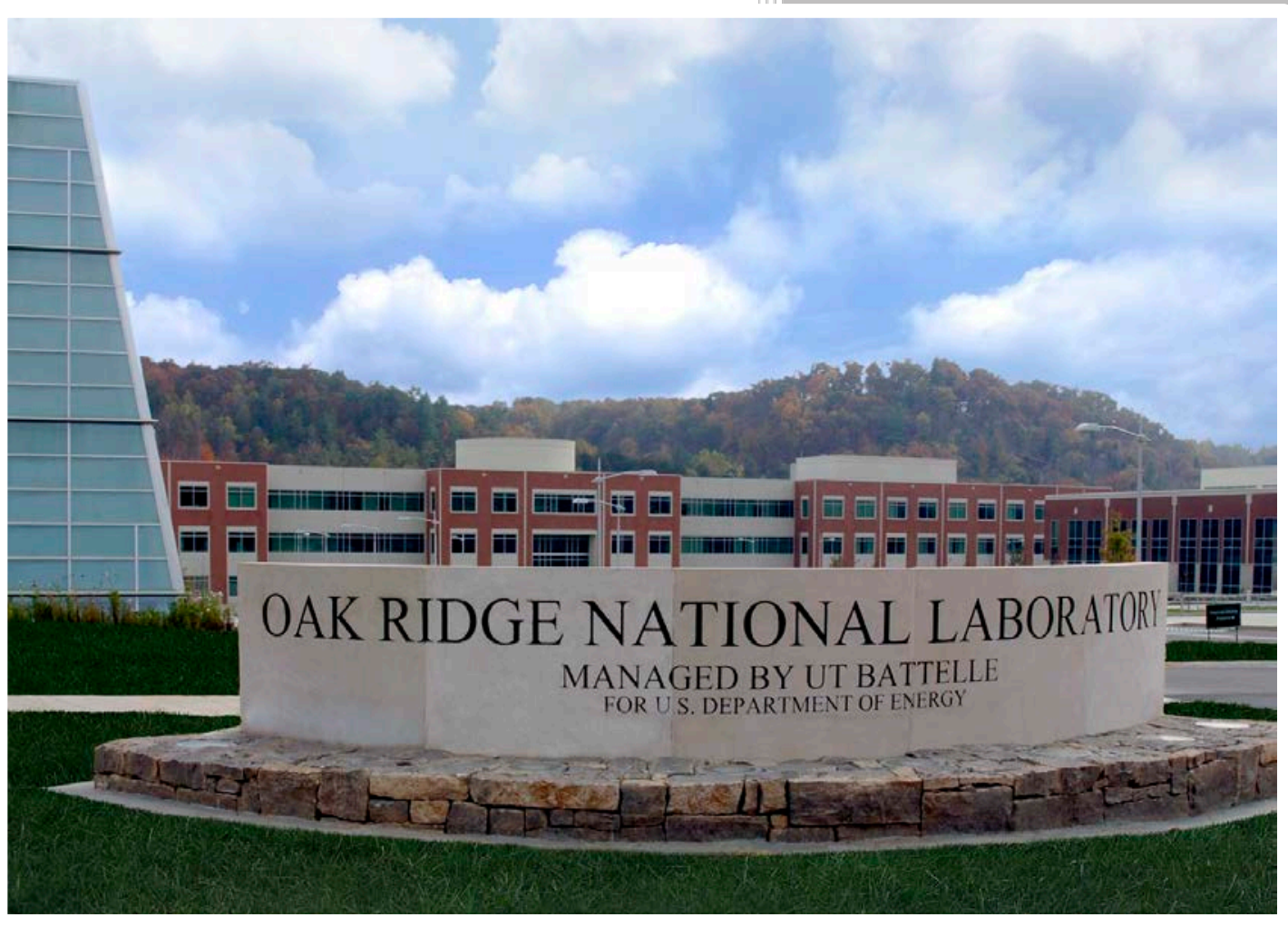

M. Parans Paranthaman

07/18/2016

CRADA FINAL REPORT

NFE-15-05761

Approved for Public Release. Distribution is Unlimited. 


\section{DOCUMENT AVAILABILITY}

Reports produced after January 1, 1996, are generally available free via US Department of Energy (DOE) SciTech Connect.

Website http://www.osti.gov/scitech/

Reports produced before January 1, 1996, may be purchased by members of the public from the following source:

National Technical Information Service

5285 Port Royal Road

Springfield, VA 22161

Telephone 703-605-6000 (1-800-553-6847)

TDD 703-487-4639

Fax 703-605-6900

E-mail info@ntis.gov

Website http://www.ntis.gov/help/ordermethods.aspx

Reports are available to DOE employees, DOE contractors, Energy Technology Data Exchange representatives, and International Nuclear Information System representatives from the following source:

Office of Scientific and Technical Information

PO Box 62

Oak Ridge, TN 37831

Telephone 865-576-8401

Fax 865-576-5728

E-mail reports@osti.gov

Website http://www.osti.gov/contact.html

This report was prepared as an account of work sponsored by an agency of the United States Government. Neither the United States Government nor any agency thereof, nor any of their employees, makes any warranty, express or implied, or assumes any legal liability or responsibility for the accuracy, completeness, or usefulness of any information, apparatus, product, or process disclosed, or represents that its use would not infringe privately owned rights. Reference herein to any specific commercial product, process, or service by trade name, trademark, manufacturer, or otherwise, does not necessarily constitute or imply its endorsement, recommendation, or favoring by the United States Government or any agency thereof. The views and opinions of authors expressed herein do not necessarily state or reflect those of the United States Government or any agency thereof. 
ORNL/TM-2016/331

CRADA/NFE-15-05761

Chemical Sciences Division

Advanced Manufacturing Office

\title{
Additive Manufacturing of Isotropic NdFeB Bonded Permanent Magnets
}

M. Parans Paranthaman ${ }^{1}$, Ling $\mathrm{Li}^{1}$, Orlando Rios ${ }^{1}$, Amy M. Elliott ${ }^{1}$, Brian Post ${ }^{1}$, Vlastimil Kunc ${ }^{1}$, Robert Fredette ${ }^{2}$, and John Ormerod ${ }^{2}$

${ }^{1}$ Oak Ridge National Laboratory, Oak Ridge, TN 37831, USA

${ }^{2}$ Magnet Applications, Inc., DuBois, PA 15801, USA

Date Published: 07/18/2016

\author{
Prepared by \\ OAK RIDGE NATIONAL LABORATORY \\ Oak Ridge, Tennessee 37831-6283 \\ managed by \\ UT-BATTELLE, LLC \\ for the \\ US DEPARTMENT OF ENERGY \\ under contract DE-AC05-00OR22725
}

This report does not contain Protected CRADA information

Approved for Public Release 


\section{CONTENTS}

\section{Page}

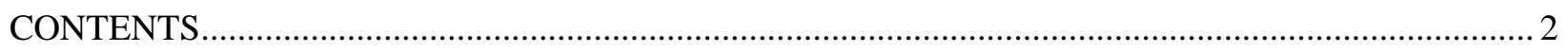

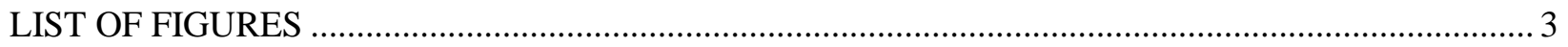

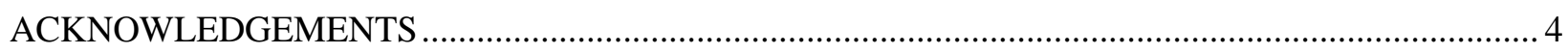

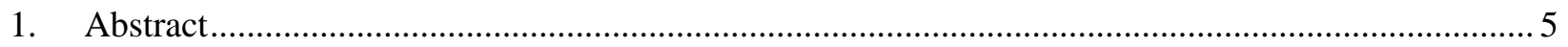

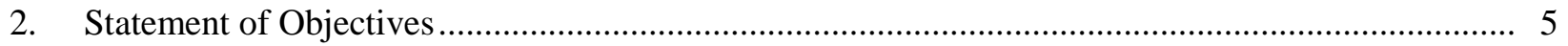

3. Benefits to the Funding DOE Office's Mission …......................................................................... 5

4. Technical Discussion of Work Performed by All Parties .............................................................. 6

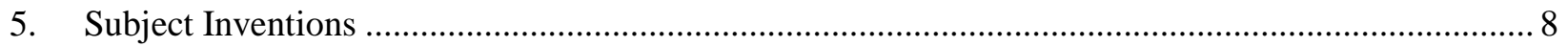

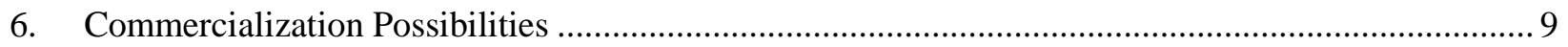

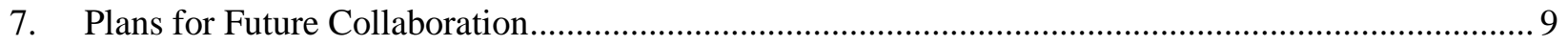

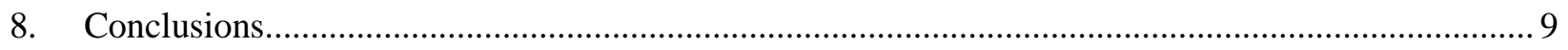

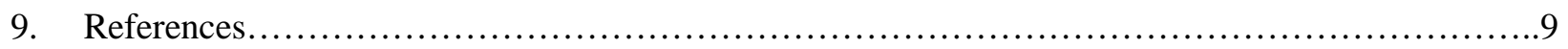




\section{LIST OF FIGURES}

Fig. 1. Images of 1” x 1” square and 1” ring near-net shape binder jet magnets after a clear polyurethane coat compared with nickel coated commercially sintered NdFeB magnets [1].

Fig. 2. Hysteresis loops of the binder jet printed ring and square magnets [1].

Fig. 3. Schematics (a) Image of the nozzle depositing layers of magnetic materials on the print bed;

(b) Schematic of the melt and extrude process, right underneath the nozzle is a printed magnet in a hollow cylinder shape with an OD $x$ ID of $\sim 4.5$ inch $x 3$ inch [2].

Fig. 4. Hysteresis loops of the BAAM printed NdFeB nylon composite magnet [2]. 


\section{ACKNOWLEDGEMENTS}

This CRADA NFE-15-05761 was conducted as a Technical Collaboration project within the Oak Ridge National Laboratory (ORNL) Manufacturing Demonstration Facility (MDF) sponsored by the US Department of Energy Advanced Manufacturing Office (CPS Agreement Number 24761). Opportunities for MDF technical collaborations are listed in the announcement "Manufacturing Demonstration Facility Technology Collaborations for US Manufacturers in Advanced Manufacturing and Materials Technologies” posted at http://web.ornl.gov/sci/manufacturing/docs/FBO-ORNL-MDF-2013-2.pdf. The goal of technical collaborations is to engage industry partners to participate in short-term, collaborative projects within the Manufacturing Demonstration Facility (MDF) to assess applicability and of new energy efficient manufacturing technologies. Research sponsored by the U.S. Department of Energy, Office of Energy Efficiency and Renewable Energy, Advanced Manufacturing Office, under contract DEAC05-00OR22725 with UT-Battelle, LLC.

Notice: This manuscript has been authored by UT-Battelle, LLC under Contract No. DE-AC0500OR22725 with the U.S. Department of Energy. The United States Government retains and the publisher, by accepting the article for publication, acknowledges that the United States Government retains a non-exclusive, paid-up, irrevocable, world-wide license to publish or reproduce the published

form of this manuscript, or allow others to do so, for United States Government purposes. The Department of Energy will provide public access to these results of federally sponsored research in accordance with the DOE Public Access Plan (http://energy.gov/downloads/doe-public-access-plan). 


\section{Abstract}

The technical objective of this technical collaboration phase I proposal is to fabricate net shape isotropic NdFeB bonded magnets utilizing additive manufacturing technologies at the ORNL MDF. The goal is to form complex shapes of thermoplastic and/or thermoset bonded magnets without expensive tooling and with minimal wasted material. Two additive manufacturing methods; the binder jet process; and big area additive manufacturing (BAAM) were used. Binder jetting produced magnets with the measured density of the magnet of $3.47 \mathrm{~g} / \mathrm{cm}^{3}$, close to $46 \%$ relative to the $N d F e B$ single crystal density of $7.6 \mathrm{~g} / \mathrm{cm}^{3}$ were demonstrated. Magnetic measurements indicate that there is no degradation in the magnetic properties. In addition, BAAM was used to fabricate isotropic near-net-shape NdFeB bonded magnets with magnetic and mechanical properties comparable or better than those of traditional injection molded magnets. The starting polymer magnet composite pellets consist of 65 vol\% isotropic NdFeB powder and 35 vol\% polyamide (Nylon-12). The density of the final BAAM magnet product reached $4.8 \mathrm{~g} / \mathrm{cm}^{3}$, and the room temperature magnetic properties are: Intrinsic coercivity $H_{c i}=8.65 \mathrm{kOe}$, Remanence $B_{r}=5.07 \mathrm{kG}$, and energy product $(B H)_{\max }=5.47$ MGOe $\left(43.50 \mathrm{~kJ} / \mathrm{m}^{3}\right)$. This study provides a new pathway for preparing near-net shape bonded magnets for various magnetic applications.

\section{Statement of Objectives}

This phase I technical collaboration project (MDF-TC-2016-79) was started on July 20, 2015 and was completed on July 20, 2016. The collaboration partner, Magnet Applications Inc. (MAI) is a small business and has established itself as an industry-leading manufacturer of compression bonded and injection molded magnets and assemblies as well as supplying the full range of permanent magnets. The company, headquartered in DuBois, PA, has sales offices across North America and is a wholly owned subsidiary of the Bunting Magnetics Company (Bunting). Magnet Applications is the largest manufacturer of compression bonded rare earth magnets and related assemblies in North America. The main objective of this project is to use additive manufacturing technologies to fabricate net shape, $25 \mathrm{~mm}$ diameter bonded NdFeB magnets with volume fraction loadings of $>60 \%$ for the thermoplastic binder. The objective is to at a minimum produce magnetic characteristics equivalent to commercially available injection molded and compression bonded $\mathrm{NdFeB}$ magnets. The target is to achieve $40 \%$ fully dense sintered magnet properties. The mechanical, magnetic, and microstructural properties of the magnets have been characterized and fully evaluated.

\section{Benefits to the Funding DOE Office's Mission}

In the 21st century, the use of permanent magnets in green energy technologies has skyrocketed. Rareearth based permanent magnets are the key components in environmentally friendly technologies such as electric vehicles and wind turbines. However, high cost of rare-earth magnets and issues with reliability of clean technology devices needs to be resolved in the upcoming years. The high cost of permanent magnets is mainly a result of the scarcity of rare-earth elements. Considering that a large portion of US rare-earth magnet manufacturing capability was transferred to China in the last decade, novel techniques that avoid or minimize the use of rare-earth elements need to be developed to reduce the US vulnerability to rare-earth prices. Bonded $\mathrm{NdFeB}$ permanent magnets have grown to be an important segment of the permanent magnet market with an estimated Global market size of $\$ 750$ million. Two manufacturing method are mainly utilized to fabricate bonded NdFeB magnets; these are injection molding using a thermoplastic binder and compression bonding using a thermoset binder. Both methods require tooling that can cost up to $\$ 50,000$ for multi-cavity injection mold tooling, making it uneconomic for low/medium volume requirements. 
Conventional manufacturing methods to make permanent magnets are inefficient and generate a sizable waste. Recycling the waste material is not an easy task considering that the rare-earth elements tend to oxidize during high temperature treatment during purification processes. Therefore, alternative ways need to be explored that not only require less energy during manufacturing but also generate less waste material. This project has identified two potential additive manufacturing methods that can be used to print magnets. The potential impacts of research include: developing net-shape magnets without tooling drastically reducing the required manufacturing investment, and improving the yield of rare earth elements (REE); and Critical Materials Institute (CMI) hub additive manufacturing project will benefit from this demonstration. Overall, the advancement of the utilization of the AM techniques could lead to the adoption of AM technology by magnet manufacturing companies and lead to higher US global manufacturing competitiveness.

\section{Technical Discussion of Work Performed by All Parties}

The binder jetting process works similarly to an ink-jet printer. A print-head passes over a bed of NdFeB powder and deposits a polymer binding agent to bind the layer of particles together. The bound powder then is coated with another layer of powder, building the desired shape in successive layers of bonded powder. Upon completion, the green part and surrounding powders are placed in an oven at temperatures between $100-150{ }^{\circ} \mathrm{C}$ for 3-6 h to cure the binder. After curing, the excess powder can be brushed away to reveal the completed "green" part. Green magnet parts were then infiltrated with a clear urethane resin to achieve the measured density of the magnet of $3.47 \mathrm{~g} / \mathrm{cm}^{3}$ close to $46 \%$ relative to the NdFeB single crystal density of $7.6 \mathrm{~g} / \mathrm{cm}^{3}$. Fig. 1 shows a comparison between the binder jet magnets and the sintered NdFeB magnets.

As shown in Fig. 1, near net-shape magnets were successfully printed. Phase composition, microstructure, and magnetic flux were measured before and after printing to determine the effect of binder jetting on these properties. Results indicate that there was deterioration in the magnetic properties after printing. The measured density of the whole binder jet square magnet piece was $3.47 \mathrm{~g} / \mathrm{cm}^{3}$. This corresponds to 46 volume $\%$ of the $\mathrm{NdFeB}$ density of $7.6 \mathrm{~g} / \mathrm{cm}^{3}$. The field dependence flux densities for the whole binder jet ring and square magnet pieces are shown in Fig. 2. Figure 2 indicates that the remanence of the binder jet magnet samples is about $0.3 \mathrm{~T}$. This compares to the remanence values of $0.5 \mathrm{~T}$ and $0.65 \mathrm{~T}$ for standard production injection molded and compression bonded isotropic NdFeB magnets. The volume fraction of $\mathrm{NdFeB}$ powders in production injection molded and compression bonded $\mathrm{NdFeB}$ magnets is typically 0.65 and 0.80 respectively. Based on these values one can estimate that the volume fraction of NdFeB powder in the binder jet magnets is approximately 0.45 . This study presents a novel method to fabricate near-net shape $\mathrm{NdFeB}$ bonded magnets through a binder jetting AM process. The long-term challenge for bonded magnets is to further densify using bimodal starting particles and/or infiltrate nanoparticles/micron size particles into the green part. 


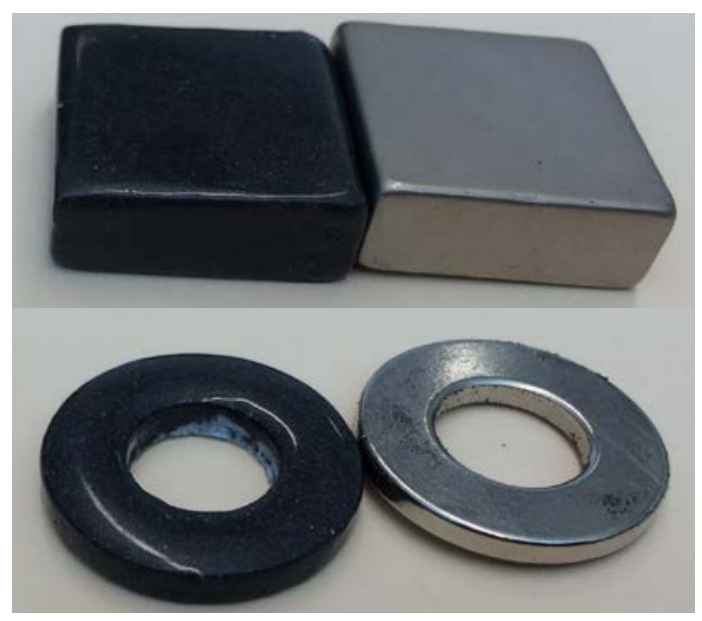

Fig. 1. Images of 1" x 1" square and 1" ring near-net shape binder jet magnets after a clear polyurethane coat compared with nickel coated commercially sintered NdFeB magnets [1].

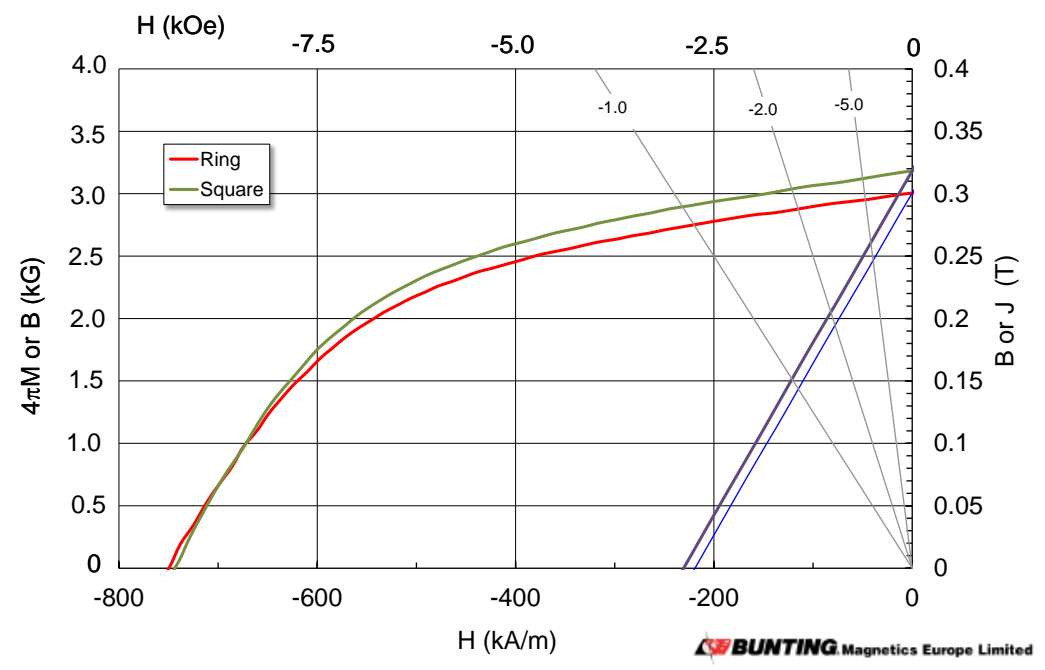

Fig. 2. Hysteresis loops of the binder jet printed ring and square magnets [1].

In addition to binder jet AM process, we also utilized the Big Area Additive Manufacturing (BAAM) system located at the MDF at ORNL to fabricate near-net-shape isotropic NdFeB bonded magnets. The BAAM system deposits high-performance engineered thermoplastics and customized thermoplastic composites via melt extrusion processing, which enables rapid manufacturing of parts completely unbounded in size. Fig. 3(a) shows the BAAM printing process of the bonded magnets: the nozzle deposits layers of magnetic materials which are fused together and solidify to form the desired shape. Instead of requiring pre-extruded filament feedstock commonly used in industry standard extrusion-based system, BAAM combines melting, compounding, and extruding functions to deposit polymer product at a controlled rate, a schematic is shown in Fig. 3(b). The feedstock materials here are magnetic pellets composed of 65 vol\% isotropic NdFeB powder (MQP-B+-10118-070) and 35 vol\% Nylon-12. It is worth mentioning that the printing of the extruded nylon magnet composite flows even better than the widely explored 3D printing plastic filament acrylonitrile butadiene styrene (ABS), and renders high accuracy. 
The magnetic, mechanical, and microstructural properties of the BAAM fabricated bonded magnets are investigated and compared with respect to the traditional injection molded commercial products made from the same starting materials. The field dependence flux densities for the BAAM magnet are shown in Fig. 4. The magnetic properties of the BAAM magnet do not show any noticeable degradation compared to those of the starting pellets. The density of the BAAM and injection molded magnets are $4.8 \mathrm{~g} / \mathrm{cm}^{3}$ and $4.9 \mathrm{~g} / \mathrm{cm}^{3}$ respectively. The BAAM magnet has the intrinsic coercivity $H_{c i}=8.65 \mathrm{kOe}$, remanence $B_{r}=$ $5.07 \mathrm{kG}$, and saturation magnetization $4 \pi M_{s} \approx 7.40 \mathrm{kG}$. The results obtained with the BAAM fabricated bonded magnets are comparable or better than those of traditional injection molded magnets.

(a)

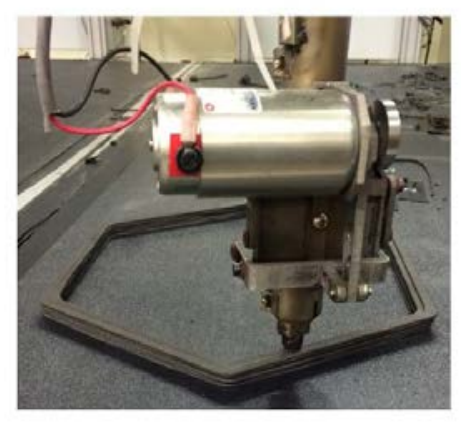

(b)

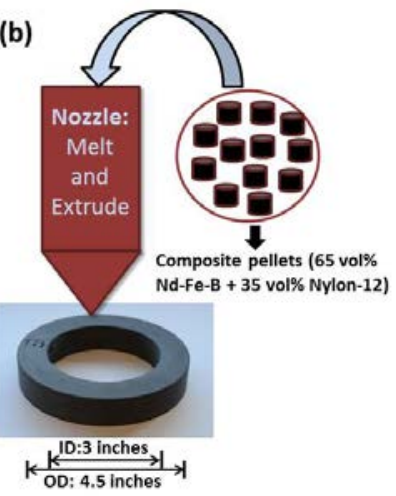

Fig. 3. Schematics (a) Image of the nozzle depositing layers of magnetic materials on the print bed; (b) Schematic of the melt and extrude process, right underneath the nozzle is a printed magnet in a hollow cylinder shape with an OD x ID of 4.5 inch x 3 inch [2].

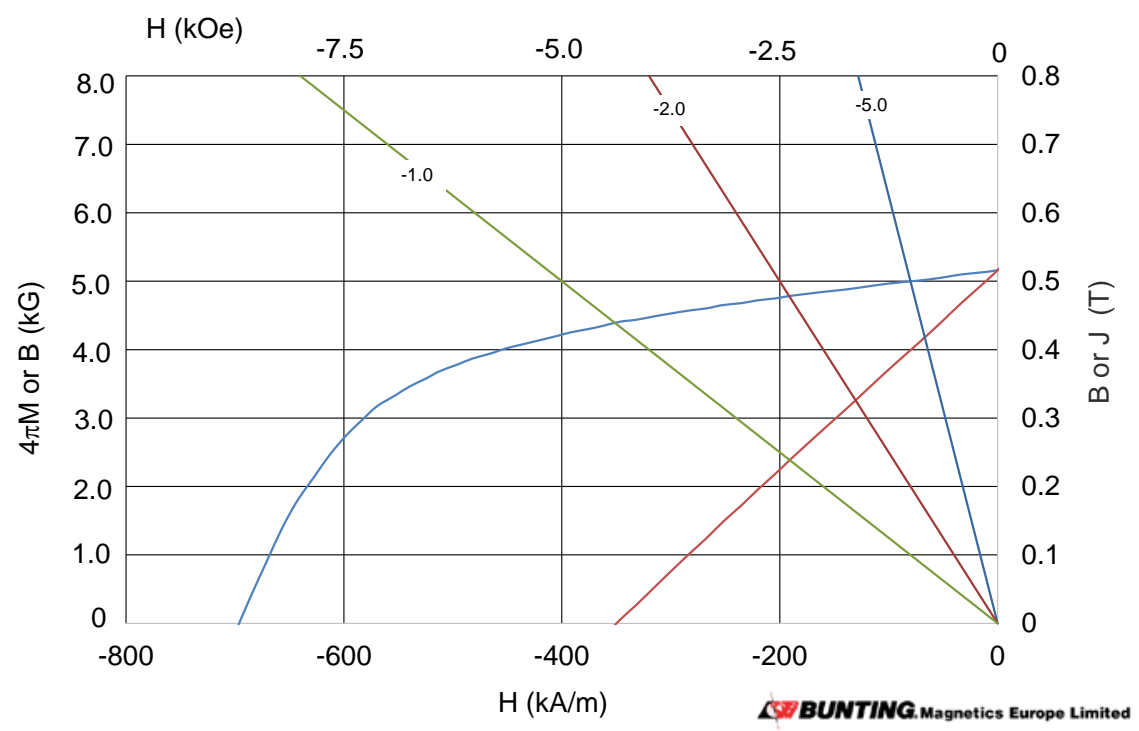

Fig. 4. Hysteresis loops of the BAAM printed NdFeB nylon composite magnet [2].

\section{Subject Inventions (As defined in the CRADA)}

Additive printing of isotropic bonded magnets via binder jetting and BAAM could be patentable. Further investigation will be needed to explore this possibility. The following two manuscripts were published based on some of the results from this research: 
1. M. Parans Paranthaman, Christopher S. Shafer, Amy M. Elliott, Derek H. Siddel, Michael A. McGuire, Robert M. Springfield, Josh Martin, Robert Fredette, and John Ormerod, "Binder Jetting: A Novel NdFeB Bonded Magnet Fabrication,” JOM, 2016, DOI: 10.1007/s11837-0161883-4.

2. Ling Li, Angelica Tirado, I.C. Nlebedim, Orlando Rios, Brian Post, Vlastimil Kun, R.R. Lowden, Edgar Lara-Curzio, Robert Fredette, John Ormerod, Thomas A. Lograsso, and M. Parans Paranthaman, "Big Area Additive Manufacturing of High Performance Bonded NdFeB Magnets,” (2016) (submitted).

\section{Commercialization Possibilities}

Bonded magnets offer a better option for making low cost intricate shapes from isotropic powder when the high magnetic performance of sintered magnets is not required. Injection molding is a wellestablished method for fabricating complex shaped bonded magnets. Here we demonstrated a novel alternate approach - Big Area Additive Manufacturing (BAAM) - to fabricate near-net-shape isotropic $\mathrm{NdFeB}$ bonded magnets. Magnetic and mechanical characterizations demonstrate that the BAAM fabricated magnets can compete with or outperform the injection molded magnets. Magnet Applications Inc. can use BAAM to fabricate bonded magnets. However, further improvements to compete with compression bonded magnets are necessary.

\section{Plans for Future Collaboration}

Additive manufacturing offers significant advantages such as cost effectiveness (no tooling required), fast speed (simple procedure), and capability of producing parts of unlimited in sizes and shapes. Therefore, BAAM provides an effective method in realizing arbitrary shape with minimum cost and waste, and has the potential to revolutionize large-scale industry production of bonded magnets. In the future work, the effect of binder type, loading fraction of the magnetic powder, anisotropic particles, and processing temperature on the magnetic and mechanical properties of the printed bonded magnets will be investigated. In addition, one of the challenges in utilizing the binder jet process to fabricate competitive magnetic performance will be the increase in volume fraction of the NdFeB powder in the magnet body. Hence, a focus of our future research will be to increase the volumetric densities of the printed magnets using bimodal distribution of particle sizes with a micron or submicron size and 70 micron size particles. Based on our success with BAAM, ORNL plan to continue working with MAI to advance BAAM technology toward the goal of achieving printed magnets with much improved magnet properties that are suitable for vast industrial magnet applications.

\section{Conclusions}

In this work, commercially-available $\mathrm{NdFeB}$ nylon polymer composite pellets were extruded through the BAAM and successfully printed magnets with properties comparable or better than that of injection molded magnets. In addition, $\mathrm{NdFeB}$ magnets were also successfully printed using a binder jet process.

To conclude, BAAM provides an effective method in realizing arbitrary shape with minimum cost and waste, and has the potential to revolutionize large-scale industry production of bonded magnets.

\section{References}


1. M. Parans Paranthaman, Christopher S. Shafer, Amy M. Elliott, Derek H. Siddel, Michael A. McGuire, Robert M. Springfield, Josh Martin, Robert Fredette, and John Ormerod, "Binder Jetting: A Novel NdFeB Bonded Magnet Fabrication,” JOM, 2016, DOI: 10.1007/s11837-0161883-4.

2. Ling Li, Angelica Tirado, I.C. Nlebedim, Orlando Rios, Brian Post, Vlastimil Kun, R.R. Lowden, Edgar Lara-Curzio, Robert Fredette, John Ormerod, Thomas A. Lograsso, and M. Parans Paranthaman, "Big Area Additive Manufacturing of High Performance Bonded NdFeB Magnets,” (2016) (submitted). 